„Neuroenhancement-Arzneimittel“ in diesem Zusammenhang aussagekräftiger wäre als der oft verwendete Begriff der „Neuroenhancement-Präparate“. NeuroenhancementArzneimittel sind Stoffe zur Beeinflussung physiologischer Funktionen durch eine pharmakologische Wirkung. Nach diesem definitorischen Teil drehte sich der Vortrag um die möglichen arzneimittelrechtlichen Grenzen.

Zunächst wurden die unmittelbar produktbezogenen Grenzen der Apothekenpflicht und Verschreibungspflicht aufgezeigt. Sodann stellte sich die Frage nach anderen, mittelbar produktbezogenen Grenzen. Eine solche kann man dem $\$ 84$ AMG entnehmen, der eine Gefährdungshaftung des pharmazeutischen Unternehmers normiert. In diesem Zusammenhang ist fraglich, ob die Verwendung von Psychopharmaka zum Neuroenhancement, also ohne Indikation, einen bestimmungsgemäßen Verbrauch i. S. des $\$ 84$ I 2 Nr. 1 AMG darstellt. Wäre dies nicht der Fall, so käme eine Haftung nicht in Frage. Was zum bestimmungsgemäßen Verbrauch gehört, wird primär durch den pharmazeutischen Unternehmer im Zulassungsdossier festgelegt. Allerdings wird von der herrschenden Meinung auch ein bestimmungsgemäßer Verbrauch angenommen, wenn der Arzneimittelgebrauch wissenschaftlich anerkannt ist und der Unternehmer trotz positiver Kenntnis oder fahrlässiger Unkenntnis keinen Ausschluss dieser Anwendung bestimmt. Der Unternehmer hat insoweit eine Beobachtungspflicht. Der Einsatz von Neuroenhancern ist allerdings nicht wissenschaftlich anerkannt, sodass er unter diesem Gesichtspunkt bestimmungswidrig ist.

Bestimmungsgemäß wäre ein Verbrauch allerdings auch, wenn ein naheliegender Fehlgebrauch objektiv vorhersehbar ist, ein Fehlgebrauch nach Expertenempfehlung erfolgt oder bei einer Propagierung der Anwendung durch den Unternehmer. All diese Fälle treffen wohl aber nicht auf das Neuroenhancement zu.

Nun stellt sich allerdings die Frage, ob hier ein sog. offlabel-use in Frage kommt. Nach der Erörterung der verschiedenen Ansichten zum off-label-use verneint Gassner dies jedoch und sieht ein Neuroenhancement durch Psychopharmaka als Arzneimittelmissbrauch. Dieser könnte nach $₫ 95$ I Nr. 1 AMG strafbar sein, wenn ein bedenkliches Arzneimittel in den Verkehr gebracht wird. Nach Gassner scheitert die Strafbarkeit jedoch schon am Inverkehrbringen, da eine entsprechende Anwendung nicht beabsichtigt ist. Auch die Bedenklichkeit des Arzneimittels wäre wohl fraglich.

Sollte es in Zukunft ein Arzneimittel mit alleiniger Neuroenhancement-Indikation geben, so stellen sich neue Fragen über die Grenzen. Verfassungsrechtlich ist aus Art. 2 I i. V. mit Art. 1 I GG ein Grundrecht auf neuronale Selbstbestimmung herzuleiten. Grenze dieses Rechts wäre der Schutz vor Selbstschädigung, welcher bei einer entsprechenden Wirkung des Mittels zu aktivieren wäre. Eine generelle Lösung scheint aber inadäquat zu sein. Daher müssen Beschränkungen aufgrund einer Betrachtung des Einzelfalles erfolgen, z.B. abgestufte Informationspflichten. Generell zulässig wären nur ,generelle Belästigungen“"wie der allseits bekannte „Zu-Risiken-und-Nebenwirkungen“-Satz am Ende einer Werbung. Der Staat muss seine Schutzpflichten vor kollektiver und individueller Fremdschädigung verhältnismäßig und einzelfallabhängig erfüllen und darf nicht zu einem , ,iberalen Paternalismus“ übergehen.

Die Diskussion begann Rosenau, welcher nach einer Unterlassenhaftung des Unternehmers fragt. Gassner verneint diese, da die Haftung des Unternehmers bereits nicht an ein Verschulden anknüpft. Ein Unterlassenstatbestand würde hier zu weit gehen. Auf Nachfrage Sprangers erklärt Gassner, dass der Unternehmer grundsätzlich ex lege bestimmt, was zum bestimmungsgemäßen Verbrauch gehört, und daher ein Einschreiten des Unternehmers, auch bei bekanntem Missbrauch in der breiten Masse, nur bei einer konkreten Gefährdung notwendig ist. Dies stelle allerdings ein Wertungsproblem dar. Hildt und Rosenau bemerken, dass die Einordnung des Neuroenhancements als Arzneimittelmissbrauch sehr negativ konnotiert, und der Begriff des off-label-use eventuell zu eng gefasst ist. Gassner weist hier auf die Schwierigkeiten der Definition hin, sieht aber einen Arzneimittelmissbrauch dann gegeben, wenn der Gebrauch nicht diagnostischen, kurativen oder lindernden Zwecken dient, was beim Neuroenhancement der Fall ist.

REZENSIONEN

DOI: $10.1007 / \mathrm{s} 00350-011-2936-x$

\section{Ludwig Ebermayer. Leben und Werk des höchsten Anklägers der Weimarer Republik unter besonderer Berücksichtigung seiner Tätigkeit im Medizin- und Strafrecht.}

Von Andreas Michael Staufer. (Leipziger Juristische Studien. Rechtshistorische Abteilung, Bd. 6), Leipziger Universitätsverlag, Leipzig 2010, 375 S., kart., €36,00

Ludwig Ebermayer (1858-1933), Senatspräsident am Reichsgericht, Oberreichsanwalt und Professor zu Leipzig in der Weimarer Republik, wirkte auch als Pionier des Medizinrechts, während es den Begriff noch nicht gab. Als produktiver Autor verfasste er eine Vielzah von Schriften, die sowohl Juristen wie Ärzte lasen, darunter die Bücher „Die zivil- und strafrechtliche Haftung des Arztes für Kunstfehler“ (1918), „Arzt und Patient in der Rechtsprechung“ (1924), „Der

Prof. Dr. iur. Dr. h.c. Adolf Laufs,

Heidelberg, Deutschland
Arzt im Recht. Rechtliches Handbuch für Ärzte“ (1930). In der „Deutschen Medizinischen Wochenschrift“ erörterte er eine Fülle von Rechtsfragen aus der ärztlichen Praxis. Bemerkenswert die thematische Breite dieses Ouevres: neben dem ärztlichen Berufs- und Standesrecht schrieb der renommierte Jurist über das Recht der Apotheken, der Heilkundigen wie -unkundigen, der Krankenkassen, der Arzneimittel und der Heilmittelwerbung, über das Krankenhaus-, das Krankenversicherungs- und das Steuerrecht, außerdem über bevölkerungspolitische Reformfragen seiner Zeit wie die Abtreibung, die Euthanasie und Sterbehilfe, die Zwangssterilisation und den Umgang mit Geschlechtskrankheiten.

Diesen medizinrechtlichen Schwerpunkt und den zweiten zum Straf- und Strafprozessrecht in einem mehr als dreihundert Einzeltitel umfassenden Gesamtwerk erschließt die vortreffliche, von Bernd-Rüdiger Kern geförderte Leipziger juristische Dissertation. Ihr Verfasser versteht es, Ebermayers Schriften in die juristischen und reformerischen Strömungen der Weimarer Zeit einzubetten und ihnen so erneut Leuchtkraft zu geben. Der in Leben und Werk Dargestellte erscheint als noble, maßvoll urteilende, die Verständigung zwischen Ärzten und Juristen suchende Persönlichkeit. Manche Fragen von Gewicht, die ihn beschäftigten, bewegen uns Heutige weiter. Auch darum verdient das Buch Staufers das Interesse der Medizinjuristen. 